\title{
Alterations of cytochrome P450-mediated drug metabolism during liver repair and regeneration after acetaminophen-induced liver injury in mice
}

Yifan Bao, Mi Phan, Junjie Zhu, Xiaochao Ma, José E. Manautou, and

Xiao-bo Zhong

Department of Pharmaceutical Sciences, School of Pharmacy, University of Connecticut, Storrs, Connecticut (Y.B., M.P., J.E.M, X-b.Z.); Department of Pharmaceutical Sciences, School of Pharmacy, University of Pittsburgh, Pittsburgh, Pennsylvania (J.Z., X.M.) 
DMD-AR-2021-000459R1

Running Title: Alterations of P450s during liver regeneration by AILI

Corresponding author: Dr. Xiao-bo Zhong, Department of Pharmaceutical Sciences, School of Pharmacy, University of Connecticut, $69 \mathrm{~N}$ Eagleville Road, Storrs, Connecticut 06269, USA. Telephone: 860-486-3697; Fax: 860-486-5792; E-mail: xiaobo.zhong@uconn.edu

Number of text pages: 37

Number of figures: 4

Number of tables: 1

Number of references: 35

Number of words in Abstract: 247

Number of words in Introduction: 773

Number of words in Discussion: 1744 


\section{ABBREVIATIONS:}

ADRs: adverse drug reactions; AILI, acetaminophen-induced liver injury; ALT, alanine aminotransferase; ANOVA, one-way analysis of variance; APAP, acetaminophen; AST, aspartate aminotransferase; BSA, bovine serum albumin; $\mathrm{CHZ}$, chlorzoxazone; CYPs: cytochrome P450s; DILI, drug-induced liver injury; EFV, efavirenz; GAPDH, glyceraldehyde-3-phosphate dehydrogenase; H\&E, hematoxylin \& eosin; HCC, hepatocellular carcinoma; HNF1A-AS1/HNF1A-AS1, human hepatocyte nuclear factor 1 alpha antisense RNA 1 (gene/IncRNA); Hnf1aos1/HNF1AOS1, mouse hepatocyte nuclear factor 1 alpha opposite strand 1 (gene/IncRNA); LC-MS, liquid chromatographytandem mass spectrometry; IncRNA, long noncoding RNA; LORR, loss of righting effect; MDZ, midazolam; miRNA, microRNA; NAFLD, non-alcoholic fatty liver disease; NAPQI, $\mathrm{N}$-acetyl-para-benzo-quinone imine; PBS, phosphate-buffered saline; $\mathrm{PH}$, partial hepatectomy; RSF, resorufin; RSG, rosiglitazone; RT-PCR, real-time polymerase chain reaction; SD, standard deviation; UNL, upper limit of normal level; UPLC-QTOFMS, ultra-performance liquid chromatography-quadrupole time of flight mass spectrometry. 


\section{ABSTRACT}

Acetaminophen (APAP)-induced liver injury (AILI) is the leading cause of acute liver failure in the United States, but its impact on metabolism, therapeutic efficacy, and adverse drug reactions (ADRs) of co- and/or subsequent administered drugs are not fully investigated. The current work explored this field with a focus on the AlLI-mediated alterations of cytochrome P450 (CYP)-mediated drug metabolism. Various levels of liver injury were induced in mice by treatment with APAP at $0,200,400$, and $600 \mathrm{mg} / \mathrm{kg}$. Severity of liver damage was determined at $24,48,72$, and 96 hours by plasma levels of alanine aminotransferase (ALT), aspartate aminotransferase (AST), microRNA miR122, and tissue staining. The expression and activities of CYP3A11, 1A2, 2B10, 2C29, and 2E1 were measured. Sedation efficacy and ADRs of midazolam, a CYP3A substrate, were monitored after APAP treatment. ALT, AST, and miR122 increased at 24 hours after APAP treatment with all APAP doses, while only 200 and $400 \mathrm{mg} / \mathrm{kg}$ treated groups recovered back to normal levels at 72 and 96 hours. The expression and activity of the CYPs significantly decreased at 24 hours with all APAP doses, but only recovered back to normal at 72 and 96 hours with 200 and 400 , but not $600 \mathrm{mg} / \mathrm{kg}$ of APAP. The alterations of CYP activities resulted in altered sedation efficacy and ADRs of midazolam, which were corrected by dose justification of midazolam. Overall, this work illustrated a low-CYP expression window after AILI, which can decrease drug metabolism and negatively impact drug efficacy and ADRs. 
DMD-AR-2021-000459R1

\section{Significance Statement}

The data generated in the mouse model demonstrated that expression and activities of CYP enzymes and correlated drug efficacy and ADRs are altered during the time course of liver repair and regeneration after liver is injured by treatment with APAP. Dose justifications based on predicted changes of CYP activities can achieve desired therapeutic efficacy and avoid ADRs. The generated data provide fundamental knowledge for translational research to drug treatment for patients during liver recovery and regeneration who have experienced AILI. 


\section{Introduction}

Acetaminophen (APAP)-induced liver injury (AILI) is a widely-impacting health issue in the United States. Overdose of APAP is responsible for nearly $50 \%$ of all acute liver failure cases in the United States (Bernal et al., 2010). AILI is also associated with more than 78,000 emergency visits, 33,000 hospitalizations, and around 500 deaths each year in the United States (Manthripragada et al., 2011; Blieden et al., 2014).

Fortunately, the majority of AlLI patients experience a full recovery process. Accumulated studies indicate that complete recovery from AlLI takes more than 2 weeks, therefore treatment of chronic co-morbidities must continue until full recovery is achieved. Clinical manifestation of AILI includes four stages. Stage I and II are latent periods, happening at 8-12 and 12-72 hours, respectively. These periods are featured by non-specific symptoms and initial increases of biomarkers for AILI, including plasma levels of alanine aminotransferase (ALT) and aspartate aminotransferase (AST) (Bunchorntavakul and Reddy, 2013). Stage III occurs between 72 and 96 hours after toxic APAP ingestion and is characterized by vomiting and symptoms of liver failure. Plasma biomarkers of AILI also reach peak values at this stage (Singer et al., 1995; Bunchorntavakul and Reddy, 2013). Stage IV occurs 5 days after APAP poisoning (Yoon et al., 2016). Biomarkers of AILI drop back to normal for patients without liver failure. Depending on the dose of APAP, stage IV can last between 14 to 28 days (Watkins et al., 2006; Bunchorntavakul and Reddy, 2013). The duration of recovery of AILI makes co-administration of drugs for controlling pre-existing chronic diseases, such as diabetes, hypertension, and depression, unavoidable. However, the alterations in 
efficacy and occurrence of adverse drug reactions (ADRs) by drug treatment for preexisting conditions during recovery from AILI are not fully known.

One of the major factors causing alterations of efficacy and ADRs is the expression and activities of drug-metabolizing enzymes, including cytochrome P450s (CYPs). CYPs are the major drug metabolizing enzymes in liver, intestine, and kidney, responsible for metabolizing over $60 \%$ of prescription drugs. Decreases of CYP functions can lead to lower plasma concentrations of active metabolites or accumulation of parental drugs, which therefore contributes to either reduced drug efficacy or increased ADRs. For example, midazolam (MDZ), a wildly used anesthetic drug, is metabolized by CYP3A4. Therefore, decreased CYP3A4 expression and function will result in increased length of anesthesia and greater risk of ADRs, including renal or hepatic dysfunction, respiratory depression, and even death (Nordt and Clark, 1997). As a result, dose adjustment is needed to achieve optimized anesthesia time and avoid ADRs. A previous study observed immediate decreases of CYPs by AILI in a dosedependent manner in mice (Bao et al., 2020). However, how CYP-mediated drug metabolism is altered during recovery from AILI is not clear. The current study examined alterations of expression and enzyme activities of several CYPs, including CYP3A11, 1A2, 2B10, 2C29, and 2E1, in mouse liver during the whole recovery process of AILI induced by different doses of APAP. Efficacy of anesthesia and ADRs of MDZ were also examined.

Because of the impact of CYPs on drug efficacy and ADRs risk, biomarkers for predicting alterations of CYPs during the recovery process of AILI are required to further guidance in dose adjustment recommendation in the clinic. Considering that the majority 
of hepatocyte loss by AILI is confined primarily to Zone 3, where high levels of CYP expression is localized, we also investigated whether biomarkers of AILI can help predict the magnitude of alterations in CYP-mediated drug metabolism during the recovery process. For this purpose, we examined traditional biomarkers, such as plasma levels of ALT and AST, and the novel biomarker, miR122 (Wang et al., 2009; Park et al., 2016; Thulin et al., 2017). Correlations between hepatic expression and activities of CYPs and plasma levels of ALT, AST, and miR122 in mice were determined in all liver and blood samples.

Complex regulation networks during liver regeneration are involved in the regulation of CYPs, where one of the important categories is long noncoding RNAs (IncRNAs). Hepatocyte nuclear factor 1 alpha antisense RNA 1 (HNF1A-AS1) is a IncRNA mainly expressed in the gastrointestinal tract, including liver, intestine, and stomach (Chen et al., 2020a). HNF1A-AS1 is involved in the regulatory network of CYPs (Chen et al., 2018) and contributes to susceptibility to APAP-induced cytotoxicity in HepaRG cells (Chen et al., 2020b). Moreover, a role for HNF1A-AS1 is also found in promoting hepatocellular carcinoma cell proliferation (Wang et al., 2017). Therefore, HNF1A-AS1 might have a potential role in the regulation of CYPs during AILI recovery. In this experiment, the expression of mouse homologous IncRNA HNF1AOS1 during initiation and recovery of AILI and its correlations with CYPs were determined. 


\section{Materials and Methods}

Chemicals and Reagents. Phosphate-buffered saline (PBS), MDZ, resorufin (RSF), and APAP were purchased from Sigma-Aldrich (St. Louis, MO). Buffered formalin (10\%) was purchased from Fisher Science (Fair Lawn, NJ). Efavirenz (EFV) was purchased from TCl American (Portland, OR). 1-OH-MDZ and 8-OH-EFV were purchased from Toronto Research Chemicals (Ontario, Canada). Rosiglitazone (RSG), demethyl-RSG, 7-ethoxyresorufin (7-ethoxy-RSF), chlorzoxazone ( $\mathrm{CHZ})$, and 6-OH-CHZ were obtained from Cayman Chemical (Ann Arbor, MI).

Animals and Drug Treatment. C57BL/6J mice were obtained from Jackson Laboratory (Bar Harbor, ME). Mice were housed in compliance with the animal care guidelines outlined by the American Association for Animal Laboratory Sciences in the Animal Resources Facility at the University of Connecticut. All animal procedures were approved by the University of Connecticut's Institutional Animal Care and Use Committee. Given the fact that male mice are more susceptible to AlLI than female mice (Dai et al., 2006), male mice were used to investigate the role of dose-selection in AILI in the current study. In brief, 8-weeks old male mice were intraperitoneally administered with a single dose of APAP $(200,400$, or $600 \mathrm{mg} / \mathrm{kg}$ ) or PBS (vehicle control). Before dosing, mice were fasted for 16 hours, with fasting lasting for 24 hours while maintaining free access to water to avoid food-induced changes in liver genes (Gavito et al., 2016). Mice were sacrificed at $24,48,72$ or 96 hours after APAP treatment to collect blood and liver samples. A part of the liver was fixed with $10 \%$ formalin buffer for histopathological 
analysis. Remaining other liver tissues were snap frozen in liquid nitrogen and then stored at $-80^{\circ} \mathrm{C}$ for further analysis of expression and activities of CYPs.

Plasma Levels of ALT and AST. Plasma levels of ALT and AST were measured as biomarkers of hepatocellular injury with a previously described method (Bao et al., 2020).

Plasma Levels of miR122. Total RNA from plasma was isolated by using the miRNeasy kit (Qiagen) with minor modifications. In brief, $700 \mu \mathrm{l}$ of QIAzol reagent was added to $200 \mu \mathrm{l}$ of plasma sample. The sample was mixed in a tube, followed by adding $3 \mu \mathrm{l}$ of internal control miRNA, at a concentration of $0.1 \mu \mathrm{M}$ (Qiagen) and $140 \mu \mathrm{l}$ of chloroform. After mixing vigorously for $15 \mathrm{~s}$, the sample was then centrifuged at 12,000 $\mathrm{g}$ for $15 \mathrm{~min}$. The upper aqueous phase was carefully transferred to a new collection tube, and $1.5 \mathrm{vol}$ of ethanol were added. The sample was then applied directly to a silica membrane containing column and the RNA was bound and cleaned by using buffers provided by the manufacturer to remove impurities. The immobilized RNA was then collected from the membrane with buffer. The quality and quantity of the RNA was evaluated by $260 / 280$ ratio using an Agilent 2200 Tape Station from Agilent Technologies (Santa Clara, CA). The efficiency of small RNA isolation is monitored by the amount of spiked-in miRNA cel-mir-39 recovered by PCR with TaqMan probe (Applied Biosystems, CA). The RNA was reverse transcribed with a TaqMan ${ }^{\mathrm{TM}}$ MicroRNA Reverse Transcription Kit (Life Technologies, Guilford, CT) and the level of miR122 was evaluated by real-time PCR (RT-PCR) with TaqMan probes (Applied Biosystems, CA) 
Liver Tissue Histology by Hematoxylin \& Eosin Staining (H\&E Staining). Liver samples were fixed with $10 \%$ buffered formalin and kept at $4^{\circ} \mathrm{C}$ for $\mathrm{H} \& \mathrm{E}$ staining. The embedding and slicing process of H\&E staining was performed by the Connecticut Veterinary Medical Diagnostic Laboratory (http://cvmdl.uconn.edu/). Pictures were taken by a phase-contrast microscope with a $40 \times$ objective lens. The scale bars were added by Image J software.

\section{Quantification of Expression Levels of CYP mRNAs and HNF1AOS1 by RT-}

PCR. Total RNAs were isolated from frozen liver tissues using a TRIzol ${ }^{\mathrm{TM}}$ reagent (Life Technologies, Guilford,CT) according to the manufacturer's protocol. RNA concentrations were measured by a NanoDrop spectrophotometer from NanoDrop Technologies (Wilmington, DE) at $260 \mathrm{~nm}$ and RNA integrity was evaluated using an Agilent 2200 Tape Station from Agilent Technologies (Santa Clara, CA). To obtain cDNAs, $1 \mu \mathrm{g}$ of total RNAs were reversely transcribed to cDNAs using an iScript ${ }^{\mathrm{TM}}$ cDNA Synthesis kit from Bio-Rad Laboratories (Hercules, CA). RT-PCR reactions were performed using a CFX96 ${ }^{\mathrm{TM}}$ Real-Time System from Bio-Rad Laboratories (Hercules, CA) with $\mathrm{iTaq}^{\mathrm{TM}}$ Universal SYBR $®$ Green Supermix from Biorad (Hercules, CA) and primers for glyceraldehyde-3-phosphate dehydrogenase (GAPDH), CYP1A2, 2B10, 2C29, 2E1, 3A11, and HNF1AOS1 from Integrated DNA Technologies, Inc. (Coralville, IA). The primer sequences for RT-PCR are listed in Table 1. Fold changes of RNA expression in the APAP-treated group compared with the PBS control at each age were calculated using the $2^{-\Delta \Delta C t}$ method with an internal normalization to GAPDH. The values were calculated for $\log _{2}$ (fold change). 
Quantification of CYP Enzyme Activities by Mass Spectrometry (MS). S9 fractions of liver tissues were isolated by centrifugation with a previously described method (Piekos et al., 2018). To determine the enzyme activities of CYP3A11, 1A2, 2B10, 2C29, and 2E1, the reaction rates of MDZ to 1-OH-MDZ, RSF to 7-ethoxy-RSF, EFV to 8-OH-EFV, RSG to demethyl-RSG, and $\mathrm{CHZ}$ to $6-\mathrm{OH}-\mathrm{CHZ}$ were measured, respectively. CYP enzyme activities in the S9 fractions were determined by ultraperformance liquid chromatography-quadrupole time of flight MS (UPLC-QTOFMS) as previously described (Tien et al., 2015).

\section{Efficacy of MDZ for Sedation Time Measured by Loss of Righting Effect} (LORR). At 24 and 48 hours after APAP treatment, MDZ at different doses was given to mice via intraperitoneal injection. Upon injection, mice were placed on their backs carefully and recorded as sedated if they failed to completely right themselves within 30s. The time an animal fails to right itself is recorded as onset time. Animals were put on their backs every minute. The first time that an animal succeeds to right itself within $30 \mathrm{~s}$ was recorded as an awake time. The time between onset and awake time was measured as a sedation time.

Statistics. The data were presented as mean \pm standard deviation (SD). Statistical analysis was performed by one-way analysis of variance (ANOVA) with the Dunnett's post-hoc test using the GraphPad Prism 7 software program from GraphPad Software, Ln. (La Jolla, CA). A value of $p<0.05$ was considered to be statistically significant. Correlations were also analyzed by Pearson correlation coefficients using GraphPad Prism 7 software program from GraphPad Software, Ln. (La Jolla, CA). A value of $p<0.05$ was considered to be statistically significant. 


\section{DMD Fast Forward. Published on August 4, 2021 as DOI: 10.1124/dmd.121.000459}

This article has not been copyedited and formatted. The final version may differ from this version.

DMD-AR-2021-000459R1 


\section{Result}

\section{Liver Injury, Repair, and Regeneration after Treatment of APAP with Different}

Doses. The different doses of APAP selected are known to produce liver injury of various grades, from mild but recoverable (200 mg/kg APAP), severe and also recoverable (400 mg/kg APAP), to life-threatening and unrecoverable damage (600 $\mathrm{mg} / \mathrm{kg}$ APAP). Elevated levels of plasma ALT and AST enzyme activities were used to evaluate various grades of liver injury caused by different doses of APAP. Liver injury grades from 0 to 4 were assigned based on fold increases of ALT and AST in comparison to the upper limit of normal level (UNL), as described in a previous publication (Bao et al., 2020).

Plasma ALT levels reflected various grades of liver injury caused by different doses of APAP and recovery capability within 96 hours after APAP treatment. All control groups at different time points had ALT values within the normal range (approximately $50 \mathrm{U} / \mathrm{L}$; Fig. 1A blue line). When mice were treated with $200 \mathrm{mg} / \mathrm{kg}$ APAP (Fig. 1A green line), the ALT levels reached 7.3- (687.0 U/L, Grade 3), 4.3- (201.9 U/L, Grade 2), 0.4(35.6U/L, Grade 0), and 0.3-fold (23.7 U/L, Grade 0) higher values than the UNL of 94 $\mathrm{U} / \mathrm{L}$ after $24,48,72$, or 96 hours, respectively. In mice treated with a single dose of 400 mg/kg APAP (Fig. 1A red line), the ALT levels were 12.1- (1230.5 U/L, Grade 4), 4.2(400.2 U/L, Grade 2), 1.6- (150.8 U/L, Grade 1), or 0.3- (28.9 U/L, Grade 0) fold greater than the UNL at $24,48,72$, or 96 hours, respectively. When mice were treated with 600 $\mathrm{mg} / \mathrm{kg}$ APAP (Fig. 1A purple line), the ALT levels were 12.6- (1184.3 U/L, Grade 4) and 11.9-fold (1121.0 U/L Grade 4) higher than the UNL at 24 or 48 hours, respectively. No 
samples from mice treated with $600 \mathrm{mg} / \mathrm{kg}$ were collected at 72 - and 96 -hour, because all mice at this high dose died prior to these time points.

Similar results were found in plasma AST levels. Significant and dose-dependent increases of AST levels were also found at 24 hours after a single dose of APAP (Fig. 1B). The AST levels of mice treated with $200 \mathrm{mg} / \mathrm{kg}$ APAP (Fig. 1B green line) reached 5.5- (568.7 U/L, Grade 3), 2.4- (289.6 U/L, Grade 1), 0.9- (114.6U/L, Grade 0), and 0.7fold (90.6 U/L, Grade 0) higher values than the UNL of $122 \mathrm{U} / \mathrm{L}$ after 24, 48, 72, or 96 hours, respectively. In mice were treated with a single dose of $400 \mathrm{mg} / \mathrm{kg}$ APAP (Fig. 1B red line), the AST levels were 12.1- (1220.0 U/L, Grade 4), 2.7- (336.6 U/L, Grade 1), 1.5- (179.0 U/L, Grade 0), or 0.4- (47.9 U/L, Grade 0) fold greater than the UNL at 24, 48, 72, or 96 hours, respectively. When mice were treated with $600 \mathrm{mg} / \mathrm{kg} \mathrm{APAP} \mathrm{(Fig.}$ 1B purple line), the AST levels were 24.6- (3000.9 U/L, Grade 4) and 30.2-fold (3696.1 $\mathrm{U} / \mathrm{L}$ Grade 4) higher than the UNL at 24 or 48 hours, respectively. Because of a $100 \%$ fatality, no samples could be collected from mice at this dose level at either 72 or 96 hours.

Liver injury and regeneration after APAP treatment were also reflected by alterations of serum levels of miR-122. Because miR122 is liver specific, it becomes an emerging novel biomarker in plasma for the early diagnosis of AILI (Jopling, 2012). Levels of miR122 were compared to the spiked-in level of cel-39. The data were presented as a $\log _{10}$ scale. Levels of miR122 were non-detectable in the control group (Fig. $1 \mathrm{C}$ blue line), presented as $\log _{10}-22$ fold compared to the spiked-in cel-39. When mice were treated with a single dose of $200 \mathrm{mg} / \mathrm{kg}$ APAP (Fig. 1C green line), the miR122 levels were $10^{-18.1}$ and $10^{-19.5}$ folds compare to cel-39 spiked-in at 24 and 48 hours, and non- 
detectable after 48 hours. When mice were treated with a single dose of $400 \mathrm{mg} / \mathrm{kg}$ APAP (Fig. 1C red line), miR12 values were $10^{0.6}$ - and $10^{-6.8}$-fold greater compare to cel-39 spiked-in at 24 and 48 hours, and non-detectable after 48 hours. When mice were treated with $600 \mathrm{mg} / \mathrm{kg}$ APAP (Fig. 1C purple line), the miR122 levels were $10^{2.74}$ and $10^{0.95}$ folds compare to cel-39 spiked-in at 24 and 48 hours. Because mice died after 48 hours with $600 \mathrm{mg} / \mathrm{kg}$ APAP treatment, no samples could be collected for analysis past 48 hours.

The injury and recovery of liver after a single dose of APAP were also confirmed by H\&E staining of liver tissue sections. The major characteristics of AILI is hepatocellular necrosis, featured as the abruption of cell membranes and nuclei of hepatocytes around the central veins. Selected liver tissue sections from mice in the 24, 48, 72, and 96 hours after APAP dosing $(0,200,400$, and $600 \mathrm{mg} / \mathrm{kg})$ were stained with H\&E. Representative images from all APAP dose and time point groups and their time-match control (PBS treated group) are shown in Fig. 1D. No liver injury was observed in any control group at any time point (top row). Large injured areas around the central vein were found at 24 hours after all APAP doses. The injured areas then gradually decreased in 200 and $400 \mathrm{mg} / \mathrm{kg}$ groups (second and third rows). At 72 and 96 hours after 200 and $400 \mathrm{mg} / \mathrm{kg}$ APAP, respectively, the area of injury completely disappeared, indicative of full recovery. However, in mice treated with $600 \mathrm{mg} / \mathrm{kg}$, the injured areas did not decrease over times (bottom row). The severity of liver injury was scored by the Ishak System following the method described in our previous publication (Bao et al., 2020). Consistently with the ALT or AST values, the most severe tissue injury occurred at 24 hours after APAP treatment. After that time point, the hepatocellular necrosis 
areas gradually decreased back to normal at 72 and 96 hours, respectively, in the 200 and $400 \mathrm{mg} / \mathrm{kg} \mathrm{APAP-treated} \mathrm{groups.} \mathrm{For} \mathrm{instance,} \mathrm{the} \mathrm{Ishak} \mathrm{scores} \mathrm{were} \mathrm{5,} \mathrm{4,} \mathrm{3,} \mathrm{and} 0$, respectively, at 24, 48, 72, and 96 hours in the $400 \mathrm{mg} / \mathrm{kg}$ APAP treated groups, suggesting extremely severe, severe, moderate, and no liver injury at these time points, respectively. The hepatocellular necrosis areas were massive in the $600 \mathrm{mg} / \mathrm{kg}$ treated groups at both 24 and 48 hours. The Ishak scores were 5 for both 24 and 48 hours in the $600 \mathrm{mg} / \mathrm{kg}$ treated groups, indicating extremely severe injury, with no possibility of regeneration and full tissue repair. Overall, supported by the biomarkers of ALT, AST, and miR122 as well as H\&E staining of liver tissues, the severity of liver injury was observed to reach the highest level at 24 hours after a single dose of APAP. After this time point, mice treated with a recoverable dose (200 and $400 \mathrm{mg} / \mathrm{kg}$ ) underwent liver repair and regeneration, but regeneration was not observed in mice treated with a highly hepatotoxic dose of APAP (600 mg/kg).

Alterations of mRNA Expression and Enzyme Activity of CYPs in Liver and their Correlation with Plasma Levels of ALT, AST, and miR122 during Liver Injury, Repair, and Regeneration Induced by APAP. Alterations of hepatic expression of CYP3A11 mRNA during APAP liver injury, repair, and regeneration were determined by RT-PCR at 24, 48, 72, and 96 hours after treatment with 200, 400, or $600 \mathrm{mg} / \mathrm{kg}$ APAP (Fig. 2A). mRNA levels of CYP3A11 were significantly decreased at 24 hours in all APAP treated groups in comparison to control groups. In the 200 and $400 \mathrm{mg} / \mathrm{kg}$ APAP groups (green and red lines, respectively), less significant decreases of CYP3A11 mRNA expression were found at 48 and 72 hours and no decreases at 96 hours, compared to the time-matched control groups. However, CYP3A11 expression was 
further decreased at 48 hours in mice treated with a lethal dose of $600 \mathrm{mg} / \mathrm{kg} \mathrm{APAP}$ (purple line). None of the animals at the $600 \mathrm{mg} / \mathrm{kg}$ APAP dose level survived at either 72 or 96 hours.

Alterations of hepatic activities of CYP3A11 during liver injury, repair, and regeneration after AILI were determined at 24, 48, 72, and 96 hours after treatment with 200, 400, or $600 \mathrm{mg} / \mathrm{kg}$ APAP (Fig. 2B). The activities of CYP3A11 were measured using a UPLC-QTOFMS based method by calculating the formation rates of $1-\mathrm{OH}-\mathrm{MDZ}$ in S9 samples isolated from mouse liver in all groups. In agreement with mRNA levels, CYP3A11 activities were significantly decreased by APAP-induced liver injury at 24 hours. Such decrease was lower during tissue repair at 48 hours, and absent at 72 and 96 hours in mice in the 200 and $400 \mathrm{mg} / \mathrm{kg}$ APAP dose groups. By contrast, significant decreases of CYP3A11 activities were observed at all time points (24 and 48 hours) in the mice treated with $600 \mathrm{mg} / \mathrm{kg}$ APAP. A significant Pearson correlation coefficient $\left(\mathrm{r}^{2}=\right.$ $0.4445, p<0.01$ ) was found between mRNAs and enzyme activities of CYP3A11 in all liver samples (Fig. 2C).

The observed alteration patterns of CYP3A11 expression (Fig. 2A) and activities (Fig. 2B) were opposite to those observed patterns for the plasma biomarkers ALT (Fig. 1A), AST (Fig. 1B), and miR122 (Fig. 1D) during liver injury, repair and regeneration induced by APAP. To study the potential relation between these biomarkers and CYP3A11 function, correlations between hepatic mRNA levels (Fig. 2D) and enzyme activities (Fig. 2E) of CYP3A11 and plasma levels of ALT, AST, and miR122 were evaluated using Pearson correlation coefficients. The result indicated that the mRNA levels of CYP3A11 presented statistically significant negative correlations with the plasma levels of ALT $\left(r^{2}\right.$ 
$=0.6852, p<0.001)$, AST $\left(r^{2}=0.5041, p<0.01\right)$, and miR122 $\left(r^{2}=0.3584, p<0.05\right)($ Fig. 2D). Statistically significant negative correlations were also observed between enzyme activities of CYP3A11 and AILI biomarkers over the injury, repair, and recovery stages with ALT $\left(r^{2}=0.6353, p<0.001\right)$, AST $\left(r^{2}=0.4860, p<0.01\right)$, and miR122 $\left(r^{2}=0.6894\right.$, $p<0.001$ ) (Fig. 2E).

Overall, significant decreases of CYP3A11 mRNA expression and enzyme activities were observed in injured livers induced by APAP. The magnitude of the decreases was correlated with the levels of liver injury reflected by the plasma biomarkers of ALT, AST, and miR-122. During the process of liver repair and regeneration, the levels of CYP3A11 mRNA expression and enzyme activities gradually increased back to normal values. The different stages of AILI, spanning from initial injury to repair, can be predicted by the levels of plasma biomarkers.

In addition to CYP3A11, alterations of mRNA expression and enzyme activity of CYP1A2, 2B10, 2C29, and 2E1 were also found during liver Injury, repair, and regeneration induced by APAP and these alterations were similarly correlated with plasma levels of ALT, AST, and miR122.

Hepatic mRNA levels of CYP1A2 (Supplemental Fig. S1A), 2C29 (Fig. S2A), and $2 \mathrm{E} 1$ (Fig. S3A) at 24, 48, 72, and 96 hours after treatment with 200,400 , or $600 \mathrm{mg} / \mathrm{kg}$ APAP were also determined by RT-PCR. At 24 hours, mRNA levels of CYP1A2, 2C29, and 2E1 were significantly decreased in APAP treated mice, compare to the control group. Mice treated with 200 and $400 \mathrm{mg} / \mathrm{kg} \mathrm{APAP}$ (green and red lines, respectively) had less significant decreases of mRNA levels of CYP1A2, 2C29, and 2E1 at 48 and 72 hours, with no decreases detected at 96 hours in comparison to the time-matched 
control groups. However, at 48 hours after $600 \mathrm{mg} / \mathrm{kg}$ APAP, expression of CYP1A2, 2C29, and 2E1 was further decreased. No mice could survive at 72 and 96 hours at this dose level.

Hepatic activity of CYP1A2 (Supplemental Fig. S1B), 2C29 (Fig. S2B), and 2E1 (Fig. S3B) at 24, 48, 72, and 96 hours after treatment with 200, 400, or $600 \mathrm{mg} / \mathrm{kg} \mathrm{APAP}$ were also evaluated. Consistently with alterations of mRNA levels, significantly decreased levels of CYP1A2, 2C29, and 2E1 activities were found at 24 hours after AILI, whereas less decreases were found during repair at 48 hours, and no decreases at 72 or 96 hours during regeneration time in the groups with 200 and $400 \mathrm{mg} / \mathrm{kg}$ APAP. In the mice treated with $600 \mathrm{mg} / \mathrm{kg}$ APAP, significant decreases of CYP1A2, 2C29, and 2E1 activities were also observed in all time points (24 and 48 hours). Levels of mRNAs and enzyme activities of CYP1A2, 2C29, and 2E1 were significant correlated in all liver samples (Supplemental Fig. S1C, S2C, and S3C).

Similar to the observed changes in CYP3A11, expression and activities of CYP1A2, $2 \mathrm{C} 29$, and $2 \mathrm{E} 1$ were opposite to levels of the plasma biomarkers of ALT, AST, and miR122 during the liver injury, repair, and regeneration induced by APAP. Significant negative correlations were presented between mRNA levels of CYP1A2, 2C29, and 2E1 and the plasma levels of ALT, AST, and miR122 (Supplemental Fig. S1D, S2D, and S3D). There were also statistically significant negative correlations between the enzyme activities of CYP1A2, 2C29, and 2E1 and the AILI biomarkers of ALT, AST, and miR122 over the injury, repair, and recovery process (Supplemental Fig. S1E, S2E, and S3E).

In sum, significantly decreased mRNA expression and enzyme activities of CYP1A2, 2C29, and 2E1 were observed in AILI. These decreased levels were correlated with the 
severity of liver injury reflected by the plasma biomarkers, including ALT, AST, and miR122. The mRNA expression and enzyme activities of CYP1A2, 2C29, and 2E1 gradually increase back to normal levels during liver repair and regeneration.

The impact of the different doses of APAP on hepatic mRNA levels of CYP2B10 were also evaluated at 24, 48, 72, and 96 hours by RT-PCR (Fig. S4A). Expression of CYP2B10 mRNA was not significant altered in any of the APAP groups compared to the control groups. Mice treated with 200 or $600 \mathrm{mg} / \mathrm{kg} \mathrm{APAP} \mathrm{(green} \mathrm{and} \mathrm{purple} \mathrm{lines,}$ respectively) presented a trend of decreases of CYP2B10 mRNA but this was not statistically significant. Moreover, significant increased expression of CYP2B10 were observed at 72 and 96 hours after APAP treatment.

Hepatic activities of CYP2B10 were also determined at 24, 48, 72, and 96 hours after APAP treatment $(200,400$, or $600 \mathrm{mg} / \mathrm{kg})$ by UPLC-QTOFMS (Fig. S4B). Unlike mRNA levels, in the $400 \mathrm{mg} / \mathrm{kg}$ APAP group, where liver regeneration and recovery occurs, significant down regulation of CYP2B10 activities were found at 24 hours after AlLI, while less decrease is found during the repair stage at 48 hours. Decreases at in CYP2B10 activity were no longer observed 72 and 96 hours after APAP treatment. The mice treated with $600 \mathrm{mg} / \mathrm{kg}$ APAP had significant decreases of CYP2B10 activities at 24 and 48 hours. Levels of mRNAs and enzyme activities of CYP2B10 were not correlated (Pearson correlation coefficient $r^{2}=0.0020, p>0.05$ ) in all liver samples (Fig. S4C).

Activities (Fig. S4E), but not expression (Fig. S4D) of CYP2B10 were negatively correlated with levels of the plasma biomarkers of ALT, AST, and miR122 during the stages of liver injury, repair, and regeneration induced by APAP. 
In conclusion, significant decreased enzyme activities, but not mRNA expression of CYP2B10 were observed in AILI. The decreased activities of CYP2B10 were correlated with the severity of liver injury reflected by ALT, AST, and miR-122. The enzyme activities of CYP2B10 gradually increase back to normal levels over repair and regeneration stages following AlLI.

Impact on the Efficacy and ADRs of MDZ by AILI. To determine whether the alterations of CYP functions during AILI, repair, and regeneration can impact drug efficacy and risk for ADRs, MDZ was selected as a model drug. MDZ is metabolized by CYP3As in mice (Perloff et al., 2003); therefore, its efficacy on sedation time should be extended and ADRs on respiration depression and risk of death should be increased when CYP3A function is decreased. Male adult mice were treated with either PBS or a single dose of $400 \mathrm{mg} / \mathrm{kg}$ APAP, which results in severe liver injury at 24 hours but resolved after 48 hours (as shown in Fig. 1). At 24 or 48 hours after APAP treatment, the mice were received an injection of either 50 or a decreased dose of $40,37.5$, or 35 $\mathrm{mg} / \mathrm{kg}$ MDZ. Total sedation time caused by MDZ was evaluated by the duration of LORR (Fig. 3). In the control groups treated with PBS after either 24 or 48 hours, an injection with a MDZ dose at $50 \mathrm{mg} / \mathrm{kg}$ resulted in a typical LORR duration time of approximately 32 min (blue square in Fig. 3). By contrast, at 24 hours after $400 \mathrm{mg} / \mathrm{kg}$ APAP treatment (red triangle in Fig. 3), which produced the most severe liver injury and significantly decreased levels of CYP3A activities at that time point, an injection with a MDZ dose at $50 \mathrm{mg} / \mathrm{kg}$ caused all mice to have sedation times of over 16 hours, eventually resulting in $100 \%$ mortality rate $(n=3)$. At 48 hours after the APAP treatment (400 mg/kg also), which has been shown to be partially recovered to certain levels of 
liver injury and to have lesser decreases in CYP3A11 activities, dosing with MDZ at 50 $\mathrm{mg} / \mathrm{kg}$ resulted in significantly longer LORR duration time of approximately $120 \mathrm{~min}$, in comparison to the control group with the normal LORR duration of approximately $32 \mathrm{~min}$. A decreased MDZ dose of $40,37.5$, and $35 \mathrm{mg} / \mathrm{kg}$ resulted in 51,32 , and $13 \mathrm{~min}$ of LORR duration time at 48 hours after $400 \mathrm{mg} / \mathrm{kg}$ APAP treatment. As a result, a decreased dose of $37.5 \mathrm{mg} / \mathrm{kg}$ administered to APAP treatment mice, based on CYP3A11 activities, led to a similar LORR duration time of around $30 \mathrm{~min}$ as non-APAP treatment control mice receiving a MDZ dose of 50 $\mathrm{mg} / \mathrm{kg}$. These results indicate that an injured liver from toxic APAP treatment with decreased CYP activities have different capabilities to metabolize drugs and can directly impact efficacy and ADRs of drugs whose pharmacokinetics and pharmacodynamics are directly dependent on CYP function. Dose justification according to the magnitude of altered CYP activities could produce the same efficacy without altering risks for ADRs.

\section{Alterations of Expression of IncRNA HNF1AOS1 in Liver and its Correlation} with CYPs during Liver Injury, Repair, and Regeneration Induced by APAP. The Hnf1aos 1 is a mouse antisense IncRNA gene located next to the transcription factor Hnf1a gene. Its human homologous IncRNA is HNF1A-AS1, which has been proven to be involved in a transcriptional regulatory network for CYP expression and function in human liver cells (Chen et al., 2018). To evaluate its potential role in the alterations of CYP expression during AILI, its hepatic expression at multiple time points during injury, repair, and regeneration after AILI were quantified by RT-PCR (Fig. 4A). Similar to the reduced expression levels of CYPs induced by toxic APAP treatment, RNA levels of HNF1AOS1 were also significantly decreased at 24 hours at all APAP doses treated in 
comparison to the control group. In the groups with 200 and $400 \mathrm{mg} / \mathrm{kg}$ APAP (green and red lines, respectively), a less significant reduction in HNF1AOS1 expression was found at 48 hours. By 72 hours after $400 \mathrm{mg} / \mathrm{kg}$ APAP, HNF1AOS1 expression was greater than that control mice, while no differences were detected at 96 hours among all groups of mice, except for mice in the $600 \mathrm{mg} / \mathrm{Kg}$ APAP group. HNF1AOS1 expression decreased significantly on a time-dependent fashion. None of the mice survived beyond the 48 hours time point (purple line).

A Pearson correlation coefficient analysis revealed statistically significant correlations between HNF1AOS1 expression in mouse liver samples and major CYPs, including CYP3A11 $\left(r^{2}=0.4301, p<0.0001\right.$, Fig. 4B), $1 A 2\left(r^{2}=0.7186, p<0.0001\right.$, Fig. 4C), 2C29 ( $r^{2}=0.5349, p<0.0001$, Fig. 4D), 2E1 $\left(r^{2}=0.2184, p<0.001\right.$, Fig. 4E). Such correlation was not noted for $2 \mathrm{~B} 10\left(\mathrm{r}^{2}=0.00004, p=0.8849\right.$, Fig. $\left.4 \mathrm{~F}\right)$. 


\section{Discussion}

The current study aims to investigate the alterations in expression and activities of major CYP enzymes and associated drug efficacy and ADRs during liver recovery and regeneration under different intensities of liver damage by AILI. The results indicated drug metabolism mediated by CYPs recovered after AILI, and expression and activities of CYPs were also correlated with plasma levels of ALT, AST, and miR122 in mice. Major CYPs in mice corresponding to the human forms were evaluated, including CYP1A2, 2B10, 2C29, 2E1, and 3A11. These alterations are further confirmed by the altered efficacy of MDZ, a model drug whose efficacy is dependent on CYP3A activities.

Over the APAP liver injury initiation and recovery process, the expression of CYPs is closely correlated with plasma biomarkers of AILI, including ALT, AST, and mir122. The recovery process of CYP expression is also closely correlated with expression of HNF1AOS1, a IncRNA regulating expression of CYPs, indicating a potential mechanism of regulation.

In the present study, the time points of 24-, 48- and 72-, and 96-hours were selected to represent day 3-4 (stage III), over day 5 (stage IV), and over 2 weeks (complete recovery) in humans, respectively. In mice, previous studies indicated that a toxic, but not lethal dose of APAP, resulted in peak levels of ALT or AST at approximately 12-24 hours after dosing (McGill et al., 2013; Bhushan et al., 2014). The results in the current study confirmed the peak of ALT or AST elevation at 24 hours after treatment (Fig. 1A \& 1B). Clinically, peak plasma AST or AST values are usually found at 72 to 96 hours (Stage III) (Bunchorntavakul and Reddy, 2013; Yoon et al., 2016), which corresponds to 
the 24-hours in mice. In mouse models, the levels of ALT and AST were reported to drop over 48 and 72 hours after APAP dosing (Bhushan et al., 2014; Preziosi et al., 2018), which is also supported by the data in the present study. A similar recovery time course for ALT and AST values was also found in humans at 5 days after the final dose of APAP (stage IV) (Bunchorntavakul and Reddy, 2013). Moreover, between 48 to 72 hours, death happened if a lethal dose is given (Bhushan et al., 2014). These time periods in mice closely match the clinical situation at 5 days after the final APAP dose, when fatal liver failure occurs (Braeuning, 2009). In a mouse model, complete resolution of biomarkers signals occurs at 96 hours after a non-lethal dose of APAP, while in AILI patients, such full recovery is usually observed between 14 to 28 days (Singer et al., 1995; Yoon et al., 2016), depending on the doses of APAP. Therefore, based on the alterations of biomarkers, the stages of recovery after AILI in mice partly mimic the pattern in humans and timepoints are selected based on these events. More studies comparing recovery progress between mice and humans, including activation of immune cells, release of cytokines, and regeneration of hepatocytes, will further validate the stages of recovery between mice and humans.

The consequences of AlLI on expression and activities of CYPs during the recovery stages from injury were investigated in the current study. Impact on CYPs during liver regeneration was previously studied in the partial hepatectomy $(\mathrm{PH})$ mouse model (Fujino et al., 2019). However, AILI has major differences in injury and recovery patterns compared to $\mathrm{PH}$ (Clemens et al., 2019), therefore potentially giving rise to different consequences on recovery of CYP expression and function. In AILI, the injury happened in zone 3 hepatocytes of the acinus, where CYPs are highly expressed 
(Tachikawa et al., 2018), instead of the non-zonal loss of tissue in $\mathrm{PH}$. Further, a previous study indicated that AILI repressed the expression and activities of CYPs in an age-dependent manner in mice (Bao et al., 2020), but the expression of CYPs over the recovery phase was not well studied. In the present study, we found that the recovery of CYPs has a similar time-profile pattern as the regeneration of the liver. After a lethal dose of APAP (600 mg/kg), expression and activities of CYPs failed to recover (Fig. 2A and 2B, Supplemental Fig. S1A and S1B, S2A and S2B, S3A and S3B, and S4A and S4B). After a toxic but not lethal dose (200 and $400 \mathrm{mg} / \mathrm{kg}$ ), expression of CYPs recovered. CYP3A11, 1A2, and 2C29 started to recover at 24 hours (Fig. 2A, Supplemental Fig. S1A and S2A), while recovery of CYP2E1 started at 48 hours (Supplemental Fig. S3A). Completely recovery of CYP3A11 and 2C29 was found at 72 to 96 hours, while others still abnormal at 96 hours (1A2 and 2E1). The activities of all CYPs, however, were found mostly repressed at 24 hours and completely recovered at 72 hours. The repression of CYPs was further supported by the extended sedation time of MDZ at 24 and 48 hours (Fig. 3), a drug whose pharmacological deactivation is dependent on CYP3A function. The results of this study clearly show that non-lethal AILI can manifest in profound alterations in drug efficacy and safety. In fact, our results also showed that a reduced dose of MDZ in mice with AILI is required to achieve the same total sedation time as the control mice (Fig. 3) and that the normal therapeutic dose of MDZ can be lethal at specific time windows during development and recovery from AILI. Lastly, these results also indicated that the repression of CYPs persists until complete recovery from AILI is achieved. 
Although intensive effort has been made to illustrate the regeneration mechanism after AILI, the underlying mechanism of regulation of CYPs during liver regeneration has not been elucidated. Regulatory events mediating changes in CYPs expression during AILI may also be operational in promoting hepatocytes proliferation and tissue repair. Previous studies indicated that HNF1A-AS1, a IncRNA involved in hepatocyte proliferation, plays an important role in the regulation network of CYPs (Chen et al., 2018; Wang et al., 2019). HNF1AOS1 is the IncRNA in mice that corresponds to the human HNF1A-AS1 form (Wang et al., 2018). In the present study, levels of HNF1AOS1 were decreased at 24 hours after APAP treatment, which values returning back to normal at 96 hours (Fig. 4A). The pattern of alterations in HNF1AOS1 expression matched the stages of initial injury and regeneration of liver. Moreover, significant correlations were found between HNF1AOS1 and mRNA levels of CYP3A11, 1A2, 2C29, and 2E1 (Fig. 4B, 4C, 4D, and 4E). These results indicated the potential role of HNF1AOS1 in the regulation of CYPs during liver regeneration after AILI. Further experiments are required to confirm and understand the mechanism role of HNF1AOS1 in the regulation of CYPs during liver regeneration after AlLI.

DILI is the major cause of acute liver failures. AILI outweighs all other hepatotoxic drugs combined (Khandelwal et al., 2011). Severe liver failure represses the expression of CYPs which affects the efficacy of drugs dependent on CYP metabolism (Bao et al., 2020). The results regarding the sedation time of MDZ in mice showed that the interval between the APAP and MDZ treatment, the expression of CYPs, and MDZ dosages impact the efficacy and ADRs of MDZ. In adults with hepatic impairment, the dosage reduction is likely to be necessary (Trouvin et al., 1988). The current findings implicate 
that a dose adjustment may be needed to prevent overdose or unexpected prolonged effects of drugs CYP-mediated drug. An appropriate dose adjustment according to the altered expression of CYPs can help to achieve the anticipated efficacy of the drug. These experiments only tested the impact of altered CYP expression by AILI on the therapeutic efficacy and safety of MDZ. Future studies using other drugs are needed to confirm these findings and provide a better understanding of the impact of DILI on the efficacy and ADRs of drugs.

To support the evaluation and dose adjustment process, we determined the correlations between major CYPs and some AILI biomarkers, including ALT, AST, and miR122. To be more exact, the CYP expression has a negative correlation with biomarker levels; but the turning point of all of them is at 24 hours after APAP treatment. The biomarker levels started increasing at 24 hours and were recovered after 96 hours, while the CYP expression started decreasing at 24 hours and was recovered after 96 hours in mice with non-lethal doses of APAP (200 and $400 \mathrm{mg} / \mathrm{kg})$. These biomarkers have the potential to improve clinical decision-making, but the measurement and evaluation of these biomarkers is time-consuming and may not be effective as an urgent assessment (Ramachandran and Jaeschke, 2019). To develop a more effective prediction method and in order to reach the most accurate prediction, more studies and evaluations on these biomarkers are needed.

Studies regarding the toxic and lethal doses of APAP in humans are limited. The current experiment highlighted that $600 \mathrm{mg} / \mathrm{kg} \mathrm{APAP}$ was a lethal dose while 200 and $400 \mathrm{mg} / \mathrm{kg}$ led to mild and severe liver injuries in mice, respectively. A previous study determined that hepatotoxicity was likely to occur after a single overdose of $7.5-10 \mathrm{~g}$ 
APAP in adult humans, which required prompt evaluation and therapeutic intervention (Bunchorntavakul and Reddy, 2013). As discussed above, the timepoints at 24-, 48-72-, and 96-hours after APAP treatment in mice correspond to $3-4,5$, and $14-28$ days in humans. Day 3-4 is considered the critical period to evaluate AILI and CYP expression, while day $14-28$ is considered the recovery period for CYPs expression and plasma biomarkers resolution. These findings support physicians in diagnosing AlLI and adjusting doses, but the medical decision-making also has to be based on other laboratory parameters, symptoms, and factors (including age, sex, nutritional status, genetic factors, medications, and chronic liver diseases) (Bunchorntavakul and Reddy, 2013). Activities of CYPs are the important factors impacting on liver clearance in pharmacokinetics modelling. In clinic, pharmacokinetic modeling is widely applied in therapeutic drug monitoring, a clinical dosing strategy allowing personized dose adjustment (Sturkenboom et al., 2021). More pharmacokinetics/pharmacodynamics studies will be needed to achieve appropriate dose adjustment practically.

In conclusion, the expression and activation of CYPs were repressed and gradually recovered after toxic non-lethal AILI. The levels of expression of major CYPs (except CYP2B10) and activities of all CYPs are correlated with ALT, AST, and miR122. The correlation was also found between CYPs and hepatic HNF1AOS1, indicating a potential mechanism of CYP regulation. The studies in human are extremely limited. The continued experimental animal and in vitro studies can help to stimulate more clinical studies and strengthen the evidence base and directions for future research. 
DMD-AR-2021-000459R1

\section{Author contributions}

Participated in research design: Y.B, M.P, J.Z, X.M, J.E.M, X-b.Z.

Conducted experiments: Y.B, J.Z.

Performed data analysis: Y.B, X-b.Z

Wrote or contributed to the writing of the manuscript: Y.B, M.P, J.Z, X.M, J.E.M, X-b.Z. 
DMD-AR-2021-000459R1

\section{Reference}

Bao Y, Wang P, Shao X, Zhu J, Xiao J, Shi J, Zhang L, Zhu HJ, Ma X, Manautou JE, and Zhong XB (2020) Acetaminophen-Induced Liver Injury Alters Expression and Activities of Cytochrome P450 Enzymes in an Age-Dependent Manner in Mouse Liver. Drug Metab Dispos 48:326-336.

Bernal W, Auzinger G, Dhawan A, and Wendon J (2010) Acute liver failure. Lancet 376:190-201.

Bhushan B, Walesky C, Manley M, Gallagher T, Borude P, Edwards G, Monga SP, and Apte U (2014) Proregenerative signaling after acetaminophen-induced acute liver injury in mice identified using a novel incremental dose model. Am J Pathol 184:3013-3025.

Blieden M, Paramore LC, Shah D, and Ben-Joseph R (2014) A perspective on the epidemiology of acetaminophen exposure and toxicity in the United States. Expert Rev Clin Pharmacol 7:341-348.

Braeuning A (2009) Regulation of cytochrome P450 expression by Ras- and beta-catenin-dependent signaling. Curr Drug Metab 10:138-158.

Bunchorntavakul C and Reddy KR (2013) Acetaminophen-related hepatotoxicity. Clin Liver Dis 17:587607, viii.

Chen L, Bao Y, Jiang S, and Zhong XB (2020a) The Roles of Long Noncoding RNAs HNF1alpha-AS1 and HNF4alpha-AS1 in Drug Metabolism and Human Diseases. Noncoding RNA 6.

Chen L, Bao Y, Piekos SC, Zhu K, Zhang L, and Zhong XB (2018) A Transcriptional Regulatory Network Containing Nuclear Receptors and Long Noncoding RNAs Controls Basal and Drug-Induced Expression of Cytochrome P450s in HepaRG Cells. Mol Pharmacol 94:749-759.

Chen L, Wang P, Manautou JE, and Zhong XB (2020b) Knockdown of Long Noncoding RNAs Hepatocyte Nuclear Factor 1alpha Antisense RNA 1 and Hepatocyte Nuclear Factor 4alpha Antisense RNA 1 Alters Susceptibility of Acetaminophen-Induced Cytotoxicity in HepaRG Cells. Mol Pharmacol 97:278-286.

Clemens MM, McGill MR, and Apte U (2019) Mechanisms and biomarkers of liver regeneration after drug-induced liver injury. Adv Pharmacol 85:241-262.

Dai G, He L, Chou N, and Wan YJ (2006) Acetaminophen metabolism does not contribute to gender difference in its hepatotoxicity in mouse. Toxicol Sci 92:33-41.

Fujino C, Sanoh S, Tateno C, Ohta S, and Kotake Y (2019) Coordinated cytochrome P450 expression in mouse liver and intestine under different dietary conditions during liver regeneration after partial hepatectomy. Toxicol Appl Pharmacol 370:133-144.

Gavito AL, Cabello R, Suarez J, Serrano A, Pavon FJ, Vida M, Romero M, Pardo V, Bautista D, Arrabal S, Decara J, Cuesta AL, Valverde AM, Rodriguez de Fonseca F, and Baixeras E (2016) Single administration of recombinant IL- 6 restores the gene expression of lipogenic enzymes in liver of fasting IL-6-deficient mice. Br J Pharmacol 173:1070-1084.

Jopling C (2012) Liver-specific microRNA-122: Biogenesis and function. RNA Bio/ 9:137-142.

Khandelwal N, James LP, Sanders C, Larson AM, Lee WM, and Acute Liver Failure Study G (2011) Unrecognized acetaminophen toxicity as a cause of indeterminate acute liver failure. Hepatology 53:567-576.

Manthripragada AD, Zhou EH, Budnitz DS, Lovegrove MC, and Willy ME (2011) Characterization of acetaminophen overdose-related emergency department visits and hospitalizations in the United States. Pharmacoepidemiol Drug Saf 20:819-826.

McGill MR, Lebofsky M, Norris HR, Slawson MH, Bajt ML, Xie Y, Williams CD, Wilkins DG, Rollins DE, and Jaeschke $H$ (2013) Plasma and liver acetaminophen-protein adduct levels in mice after acetaminophen treatment: dose-response, mechanisms, and clinical implications. Toxicol Appl Pharmacol 269:240-249.

Nordt SP and Clark RF (1997) Midazolam: a review of therapeutic uses and toxicity. J Emerg Med 15:357365. 
Park HK, Jo W, Choi HJ, Jang S, Ryu JE, Lee HJ, Lee H, Kim H, Yu ES, and Son WC (2016) Time-course changes in the expression levels of miR-122, -155, and -21 as markers of liver cell damage, inflammation, and regeneration in acetaminophen-induced liver injury in rats. J Vet Sci 17:45-51.

Perloff MD, Von Moltke LL, and Greenblatt DJ (2003) Differential metabolism of midazolam in mouse liver and intestine microsomes: a comparison of cytochrome $\mathrm{P} 450$ activity and expression. Xenobiotica; the fate of foreign compounds in biological systems 33:365-377.

Piekos SC, Chen L, Wang P, Shi J, Yaqoob S, Zhu HJ, Ma X, and Zhong XB (2018) Consequences of Phenytoin Exposure on Hepatic Cytochrome P450 Expression during Postnatal Liver Maturation in Mice. Drug Metab Dispos 46:1241-1250.

Preziosi M, Okabe H, Poddar M, Singh S, and Monga SP (2018) Endothelial Wnts regulate beta-catenin signaling in murine liver zonation and regeneration: A sequel to the Wnt-Wnt situation. Hepatol Commun 2:845-860.

Ramachandran A and Jaeschke H (2019) Acetaminophen Hepatotoxicity. Semin Liver Dis 39:221-234.

Singer AJ, Carracio TR, and Mofenson HC (1995) The temporal profile of increased transaminase levels in patients with acetaminophen-induced liver dysfunction. Ann Emerg Med 26:49-53.

Sturkenboom MGG, Märtson A-G, Svensson EM, Sloan DJ, Dooley KE, van den Elsen SHJ, Denti P, Peloquin CA, Aarnoutse RE, and Alffenaar J-WC (2021) Population Pharmacokinetics and Bayesian Dose Adjustment to Advance TDM of Anti-TB Drugs. Clinical pharmacokinetics 60:685710.

Tachikawa M, Sumiyoshiya Y, Saigusa D, Sasaki K, Watanabe M, Uchida Y, and Terasaki T (2018) Liver Zonation Index of Drug Transporter and Metabolizing Enzyme Protein Expressions in Mouse Liver Acinus. Drug Metab Dispos 46:610-618.

Thulin P, Hornby RJ, Auli M, Nordahl G, Antoine DJ, Starkey Lewis P, Goldring CE, Park BK, Prats N, Glinghammar B, and Schuppe-Koistinen I (2017) A longitudinal assessment of miR-122 and GLDH as biomarkers of drug-induced liver injury in the rat. Biomarkers 22:461-469.

Tien YC, Liu K, Pope C, Wang P, Ma X, and Zhong XB (2015) Dose of Phenobarbital and Age of Treatment at Early Life are Two Key Factors for the Persistent Induction of Cytochrome P450 Enzymes in Adult Mouse Liver. Drug Metab Dispos 43:1938-1945.

Trouvin JH, Farinotti R, Haberer JP, Servin F, Chauvin M, and Duvaldestin P (1988) Pharmacokinetics of midazolam in anaesthetized cirrhotic patients. Br J Anaesth 60:762-767.

Wang C, Mou L, Chai HX, Wang F, Yin YZ, and Zhang XY (2017) Long non-coding RNA HNF1A-AS1 promotes hepatocellular carcinoma cell proliferation by repressing NKD1 and P21 expression. Biomed Pharmacother 89:926-932.

Wang K, Zhang S, Marzolf B, Troisch P, Brightman A, Hu Z, Hood LE, and Galas DJ (2009) Circulating microRNAs, potential biomarkers for drug-induced liver injury. Proc Natl Acad Sci U S A 106:4402-4407.

Wang Y, Xie Y, Li L, He Y, Zheng D, Yu P, Yu L, Tang L, Wang Y, and Wang Z (2018) EZH2 RIP-seq Identifies Tissue-specific Long Non-coding RNAs. Curr Gene Ther 18:275-285.

Wang Y, Yan L, Liu J, Chen S, Liu G, Nie Y, Wang P, Yang W, Chen L, Zhong X, Han S, and Zhang L (2019) The HNF1alpha-Regulated LncRNA HNF1alpha-AS1 Is Involved in the Regulation of Cytochrome P450 Expression in Human Liver Tissues and Huh7 Cells. J Pharmacol Exp Ther 368:353-362.

Watkins PB, Kaplowitz N, Slattery JT, Colonese CR, Colucci SV, Stewart PW, and Harris SC (2006) Aminotransferase elevations in healthy adults receiving 4 grams of acetaminophen daily: a randomized controlled trial. JAMA 296:87-93.

Yoon E, Babar A, Choudhary M, Kutner M, and Pyrsopoulos N (2016) Acetaminophen-Induced Hepatotoxicity: a Comprehensive Update. J Clin Transl Hepatol 4:131-142. 
DMD-AR-2021-000459R1

\section{Footnotes.}

This study was partly supported by the National Institutes of Health (NIH) National Institute of General Medical Sciences [Grant R01GM-118367 to X.Z.]; the National Institute of Allergy and Infectious Diseases [Grant R01 Al131983 to X.M.]. 
DMD-AR-2021-000459R1

\section{Financial Disclosure:}

No author has an actual or perceived conflict of interest with the contents of this article. 


\section{Figure Legends}

Fig. 1. Liver injury, repair, and regeneration after treatment with different doses of acetaminophen (APAP). Adult male mice were treated with APAP at different doses $(0$, $200,400$, or $600 \mathrm{mg} / \mathrm{kg})(\mathrm{n}=3-5)$. Blood samples and liver tissues were collected at different time points $(0,24,48,72$, or 96 hours $)$ after the APAP treatment. (A) Plasma levels of alanine aminotransferase (ALT) activity. (B) Plasma levels of aspartate aminotransferase (AST) activity. (C) Plasma levels of microRNA miR122. Ct values of miR122 are compared to that of spiked-in synthesized cel-miR-39. Data are shown as mean \pm SD and analyzed by one-way ANOVA with the Dunnett's post-hoc test. ${ }^{*} p<0.05$, ${ }^{* *} p<0.01,{ }^{* * *} p<0.001$ APAP treatment versus age-matched controls. (D) Representative images of liver sections stained with H\&E from mice treated with $0,200,400$, or 600 $\mathrm{mg} / \mathrm{kg}$ APAP at different time points. Arrows indicate the necrosis areas featured as the abruption of cell membranes and nuclei of hepatocytes around the central veins. A scale bar equals $100 \mu \mathrm{m}$.

Fig. 2. Levels of mRNA expression and enzyme activity of CYP3A11 and their correlations with plasma levels of ALT, AST, and miR122 during liver injury, repair, and regeneration induced by APAP at different doses. (A) Hepatic mRNA expression of CYP3A11 at different time points $(0,24,48,72$, or 96 hours $)$ after APAP treatment with different doses at $0,200,400$, or $600 \mathrm{mg} / \mathrm{kg}(\mathrm{n}=3-5)$. The fold changes of relative mRNA expression compared to the control mice at $0 \mathrm{~h}$ were calculated using the $2^{-\Delta \Delta t}$ method after normalization with GAPDH. (B) Enzyme activity of CYP3A11 at different 
time points $(0,24,48,72$, or 96 hours $)$ in liver S9 fraction of mice treated with 0,200 , 400, or $600 \mathrm{mg} / \mathrm{kg} \mathrm{APAP}(\mathrm{n}=3)$. The enzyme activity was determined by the formation rate of 1 -hydroxymidazolam (1-OH-MDZ) quantified by UPLC-QTOFMS. ${ }^{*} p<0.05$, ${ }^{* *} p<0.01,{ }^{* * *} p<0.001$ APAP treatment versus time-matched controls. (C) Correlation between hepatic mRNA expression of CYP3A11 and enzyme activity of CYP3A ( $n=42)$. Levels of mRNA were normalized to $0 \mathrm{mg} / \mathrm{kg}$ group at 24 hours. (D) Left panel: correlation between ALT and mRNA levels of CYP3A11 $(n=52)$. Middle panel: correlation between AST and mRNA levels of CYP3A11 ( $n=52)$. Right panel: correlation between miR122 and mRNA levels of CYP3A11 $(n=18)$. (E) Left panel: correlation between ALT and activity levels of CYP3A11 ( $n=42)$. Middle panel: correlation between AST and activity of CYP3A11 $(n=42)$. Right panel: correlation between miR122 and activity of CYP3A11 $(n=18)$.

Fig. 3. Impact of AILI on efficacy and ADRs of midazolam (MDZ). Sedation times (h) and risk of death were used to indicate efficacy and ADRs of MDZ. Adult male mice were treated with $400 \mathrm{mg} / \mathrm{kg}$ APAP or control. At 24 or 48 hours after the APAP treatment, the mice were treated with different doses of $\operatorname{MDZ}(50,40,37.5$, or $35 \mathrm{mg} / \mathrm{kg})$. Sedation times were examined by LORR. ${ }^{* * *} p<0.001$ APAP treatment versus timematched controls. 
Fig. 4. Alterations of haptic RNA expression of IncRNA HNF1AOS1 and its correlations with mRNA expression of CYPs. Liver tissues $(n=3-5)$ were collected at different time points $(0,24,48,72$ or 96 hours $)$ after the treatment with different doses of APAP $(0$, 200, 400, or $600 \mathrm{mg} / \mathrm{kg}$ ). (A) RNA levels of IncRNA HNF1AOS1. ${ }^{*} p<0.05,{ }^{* *} p<0.01$, ${ }^{* * *} p<0.001$ APAP treatment versus time-matched controls. Pearson correlations between RNA levels of HNF1AOS1 and mRNA levels of CYP3A11 (B), 1A2 (C), 2C29 (D), 2E1 (E), and 2B10 (F) $(\mathrm{n}=51)$. 
Table 1. Primer sequences used for RNA quantification by RT-PCR

\begin{tabular}{|l|l|}
\hline Gene name & Primer \\
\hline \multirow{2}{*}{ Cyp1a2 } & F: AGACAATGGCGGTCTCATC \\
& R: GACGGTCAGAAAGCCGTGGT \\
\hline \multirow{2}{*}{ Cyp2b10 } & F: TTCTGCGCATGGAGAAGGAGAAGT \\
& R: TGAGCATGAGCAGGAAGCCATAGT \\
\hline \multirow{2}{*}{ Cyp2c29 } & F: GCTCTCCTACTCCTGCTGAAGT \\
\hline \multirow{2}{*}{ Ryp2e1 1 ATGTGGCTCCTGTCTTGCATGC } \\
\hline \multirow{2}{*}{ Cyp3a11 } & F: GTCTTTAACCAAGTTGGCAA \\
\hline \multirow{2}{*}{ Gapdh } & R: CCA ATC AGA AAG GTA GGG TC \\
\hline \multirow{2}{*}{ Hnflaos1 } & R: CCAAGCAAGCAGGGATGGAC \\
& F: GGTGGAGCCAAAAGGGTCAT \\
\hline & R: GGTTCACACCCATCACAAACAT \\
\hline
\end{tabular}


A
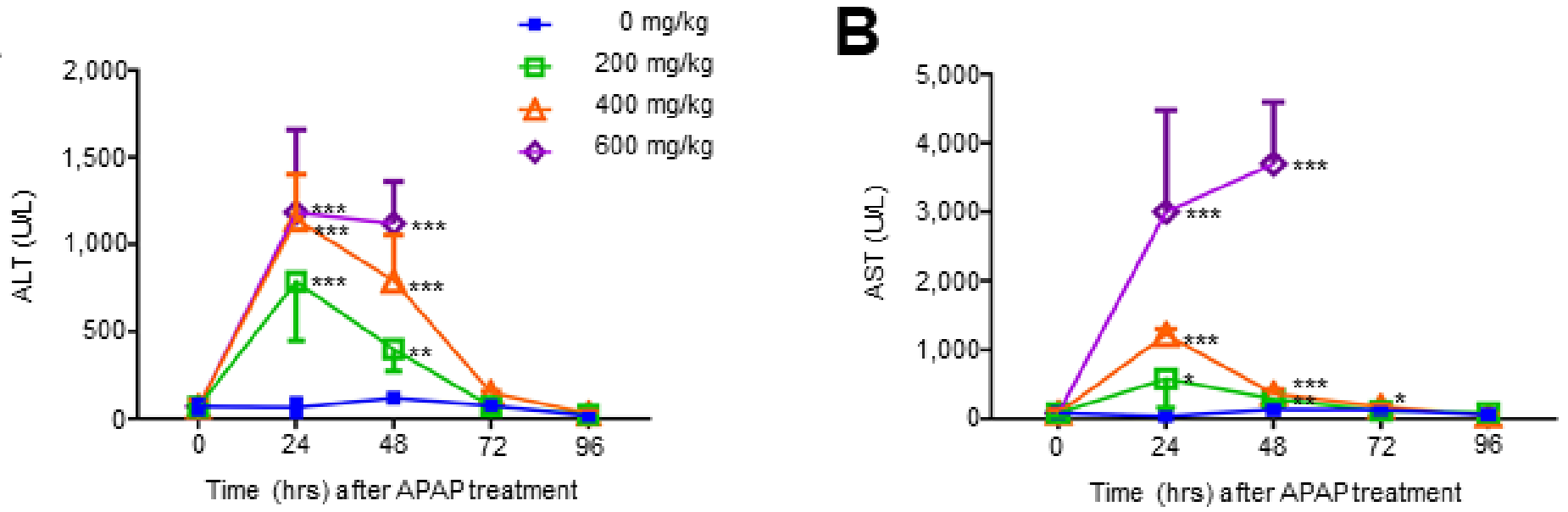

C

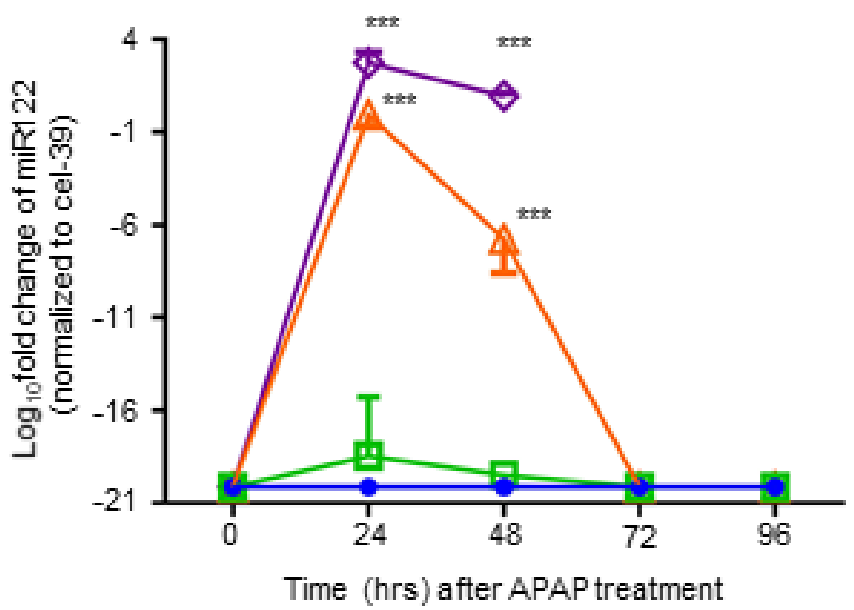

D

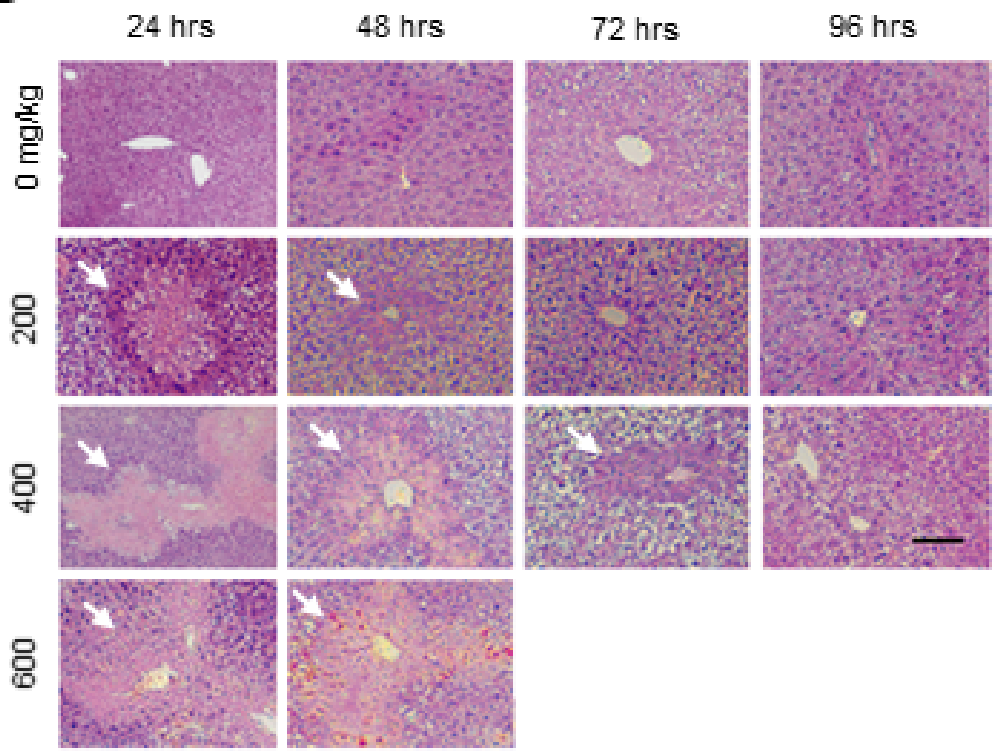

Fig. 1. Liver injury, repair, and regeneration after treatment with different doses of acetaminophen (APAP). Adult male mice were treated with APAP at different doses $(0,200$, 400 , or $600 \mathrm{mg} / \mathrm{kg})(\mathrm{n}=3-5)$. Blood samples and liver tissues were collected at different time points $(0,24,48,72$, or 96 hours) after the APAP treatment. (A) Plasma levels of alanine aminotransferase (ALT) activity. (B) Plasma levels of aspartate aminotransferase (AST) activity. (C) Plasma levels of microRNA miR122. Ct values of miR122 are compared to that of spiked-in synthesized cel-miR-39. Data are shown as mean \pm SD and analyzed by oneway ANOVA with the Dunnett's post-hoc test. ${ }^{*} p<0.05,{ }^{* *} p<0.01,{ }^{* \star *} p<0.001$ APAP treatment versus age-matched controls. (D) Representative images of liver sections stained with H\&E from mice treated with $0,200,400$, or $600 \mathrm{mg} / \mathrm{kg}$ APAP at different time points. Arrows indicate the necrosis areas featured as the abruption of cell membranes and nuclei of hepatocytes around the central veins. A scale bar equals $100 \mu \mathrm{m}$. 
DMD Fast Fory
A
This article has not beencopvedished on August 4, 2021 as DOI: $10.1124 / \mathrm{dn}$ (d.121.000459
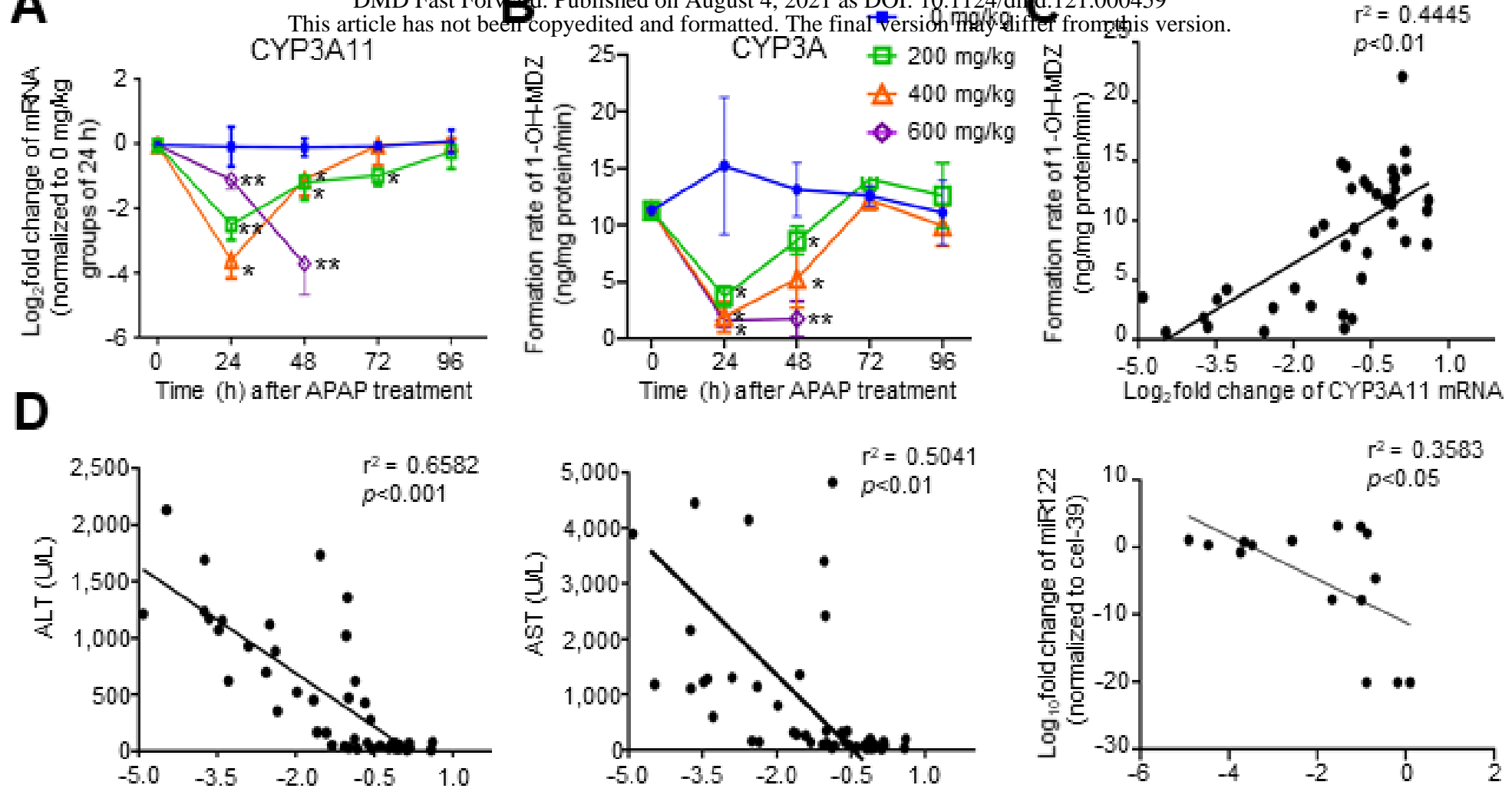

$\log _{2}$ fold change of CYP3A11 mRNA

$\log _{2}$ fold change of CYP3A11 mRNA

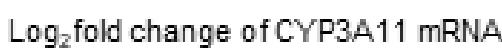

E
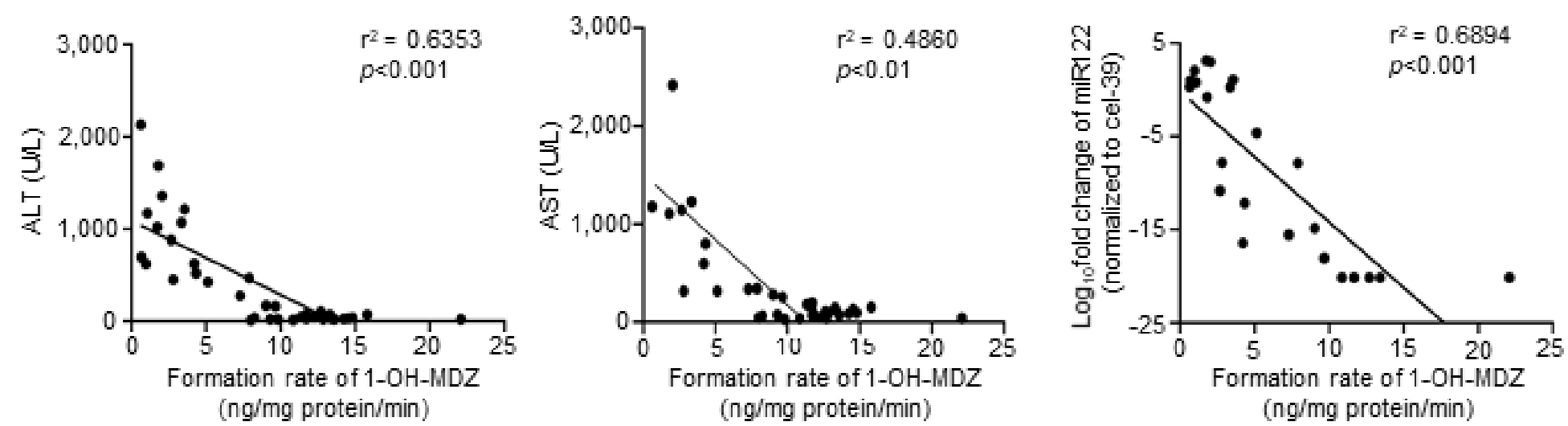

Fig. 2. Levels of mRNA expression and enzyme activity of CYP3A11 and their correlations with plasma levels of ALT, AST, and miR122 during liver injury, repair, and regeneration induced by APAP at different doses. (A) Hepatic mRNA expression of CYP3A11 at different time points (0, 24, 48,72 , or 96 hours) after APAP treatment with different doses at $0,200,400$, or $600 \mathrm{mg} / \mathrm{kg}(\mathrm{n}=3$ 5 ). The fold changes of relative mRNA expression compared to the control mice at $0 \mathrm{~h}$ were calculated using the $2^{-\Delta \Delta t}$ method after normalization with GAPDH. (B) Enzyme activity of CYP3A11 at different time points $(0,24,48,72$, or 96 hours) in liver S9 fraction of mice treated with $0,200,400$, or $600 \mathrm{mg} / \mathrm{kg} \mathrm{APAP}(\mathrm{n}=3$ ). The enzyme activity was determined by the formation rate of 1 -hydroxymidazolam (1-OH-MDZ) quantified by UPLC-QTOFMS. ${ }^{*} p<0.05$, ${ }^{* *} p<0.01,{ }^{* \star *} p<0.001$ APAP treatment versus time-matched controls. (C) Correlation between hepatic mRNA expression of CYP3A11 and enzyme activity of CYP3A ( $n=42)$. Levels of mRNA were normalized to $0 \mathrm{mg} / \mathrm{kg}$ group at 24 hours. (D) Left panel: correlation between ALT and mRNA levels of CYP3A11 ( $n=52$ ). Middle panel: correlation between AST and mRNA levels of CYP3A11 $(n=52)$. Right panel: correlation between miR122 and mRNA levels of CYP3A11 $(n=18)$. (E) Left panel: correlation between ALT and activity levels of CYP3A11 $(n=42)$. Middle panel: correlation between AST and activity of CYP3A11 $(n=42)$. Right panel: correlation between miR122 and activity of CYP3A11 $(n=18)$. 


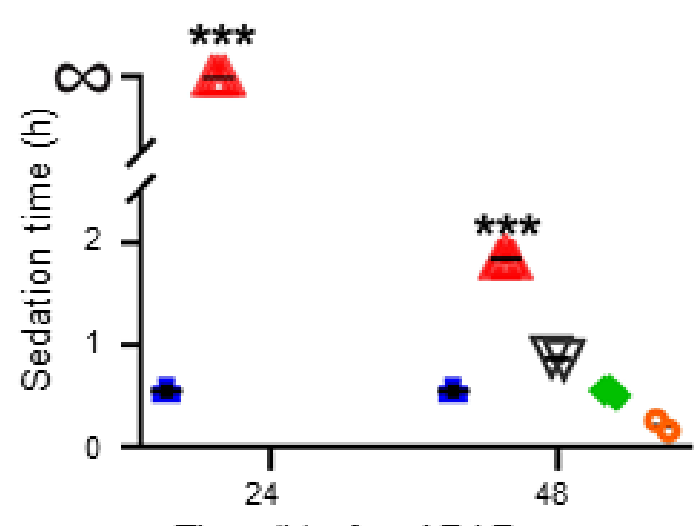

- $0 \mathrm{mg} / \mathrm{kg}$ APAP

- $+50 \mathrm{mg} / \mathrm{kg} \mathrm{MDZ}$

$400 \mathrm{mg} / \mathrm{kg}$ APAP

$\Delta+50 \mathrm{mg} / \mathrm{kg} \mathrm{MDZ}$

$\nabla \begin{aligned} & 400 \mathrm{mg} / \mathrm{kg} \text { APAP } \\ & +40 \mathrm{mg} / \mathrm{kg} \mathrm{MDZ}\end{aligned}$

$400 \mathrm{mg} / \mathrm{kg}$ APAP

$+37.5 \mathrm{mg} / \mathrm{kg} \mathrm{MDZ}$

- $400 \mathrm{mg} / \mathrm{kg}$ APAP

$+35 \mathrm{mg} / \mathrm{kg} \mathrm{MDZ}$

Time (h) after APAP treatment

Fig. 3. Impact of AlLI on efficacy and ADRs of midazolam (MDZ). Sedation times (h) and risk of death were used to indicate efficacy and ADRs of MDZ. Adult male mice were treated with 400 $\mathrm{mg} / \mathrm{kg}$ APAP or control. At 24 or 48 hours after the APAP treatment, the mice were treated with different doses of MDZ $(50,40,37.5$, or $35 \mathrm{mg} / \mathrm{kg})$. Sedation times were examined by LORR. ${ }^{* * *} p<0.001$ APAP treatment versus time-matched controls. 

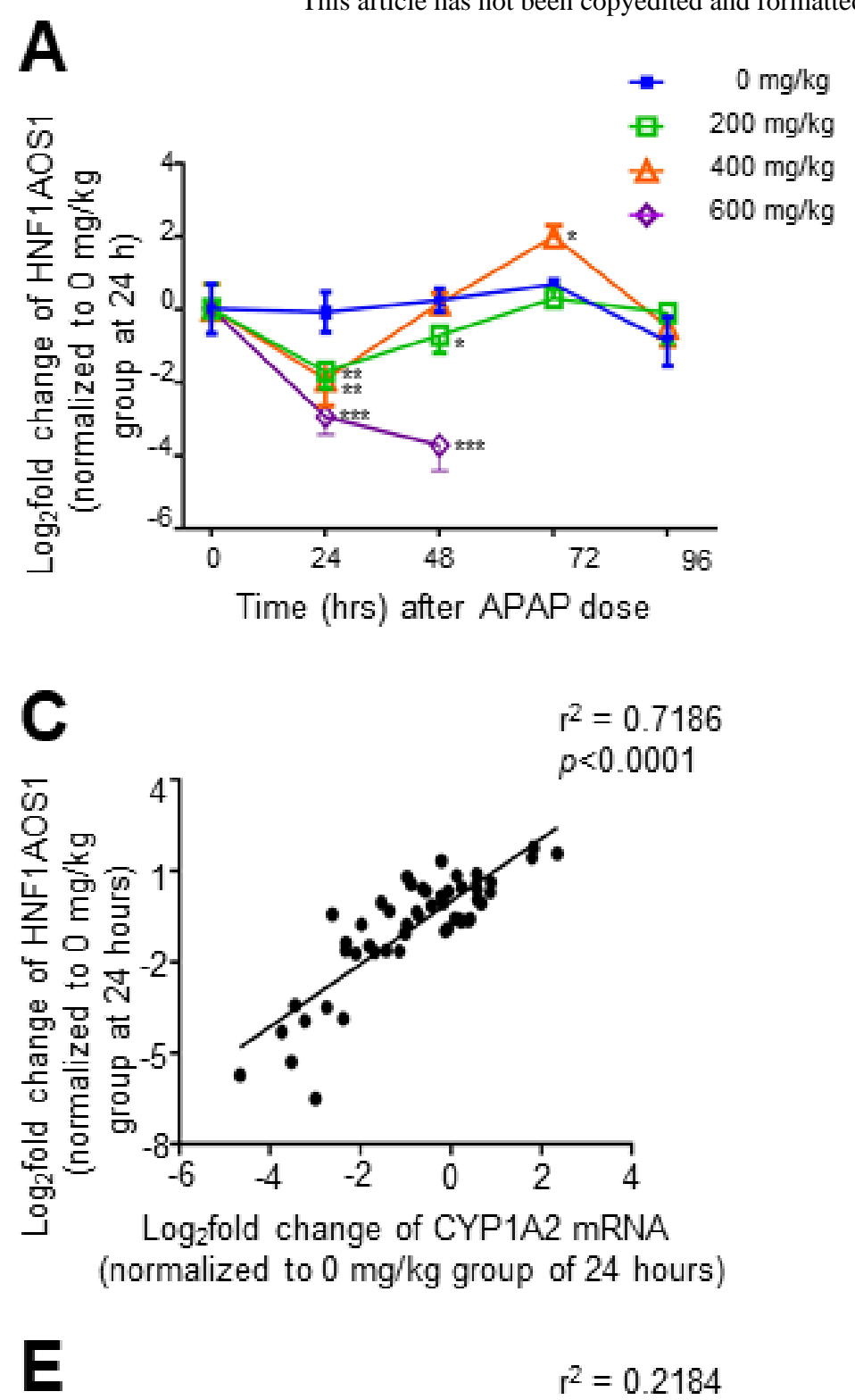

$r^{2}=0.2184$

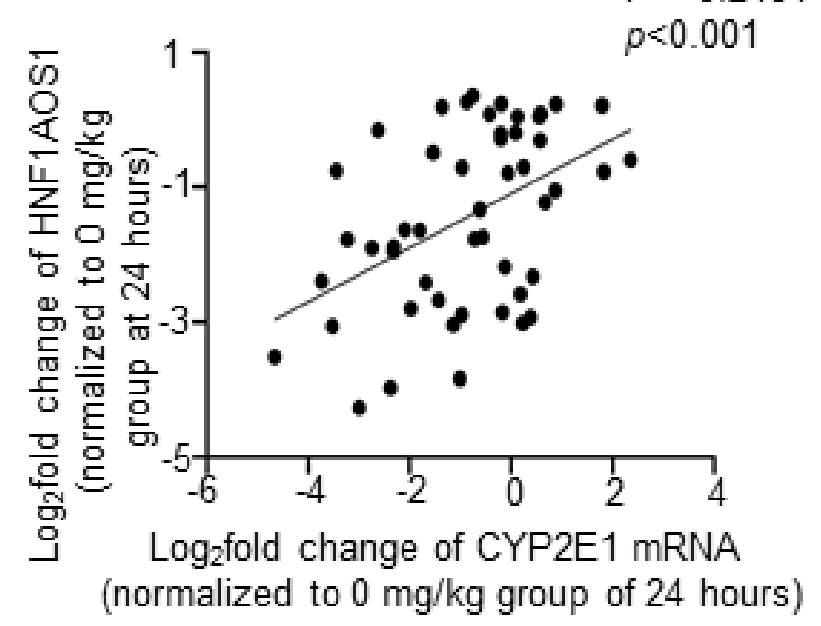

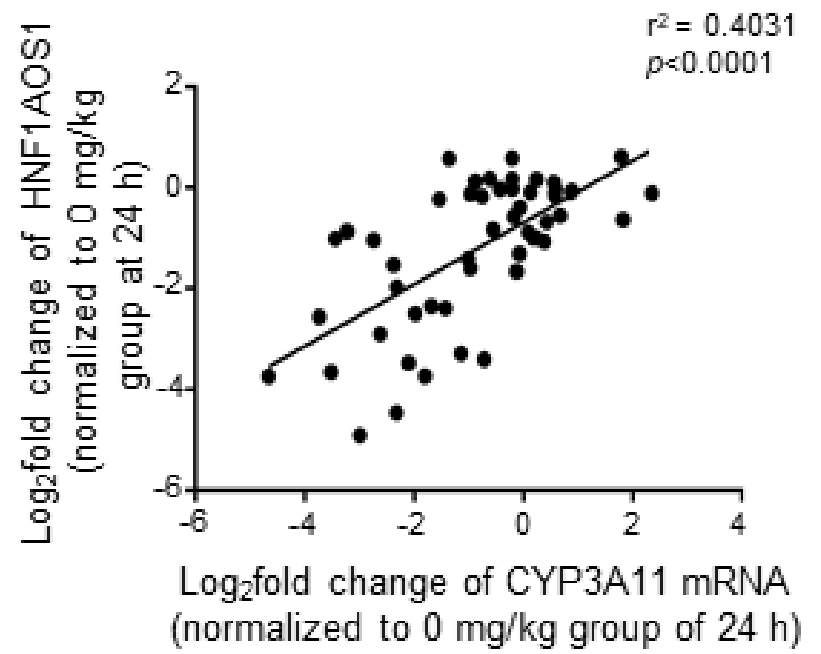

D

$r^{2}=0.5394$
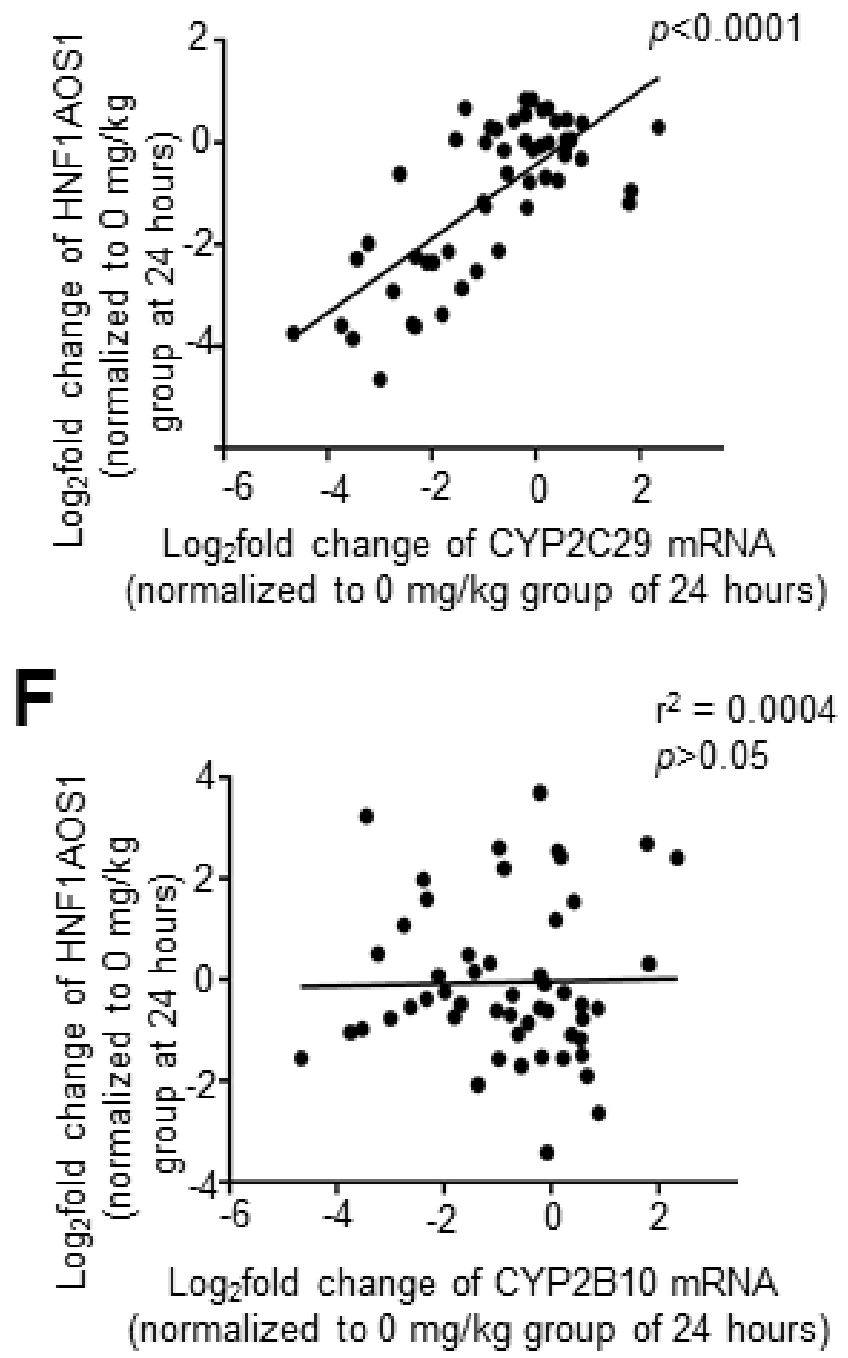

Fig. 4. Alterations of haptic RNA expression of IncRNA HNF1AOS1 and its correlations with mRNA expression of CYPs. Liver tissues $(n=3-5)$ were collected at different time points $(0,24,48,72$ or 96 hours $)$ after the treatment with different doses of $\operatorname{APAP}(0,200$, 400 , or $600 \mathrm{mg} / \mathrm{kg}$ ). (A) RNA levels of IncRNA HNF1AOS1. ${ }^{*} p<0.05,{ }^{* *} p<0.01,{ }^{* * *} p<0.001$ APAP treatment versus time-matched controls. Pearson correlations between RNA levels of HNF1AOS1 and mRNA levels of CYP3A11 (B), 1A2 (C), 2C29 (D), 2E1 (E), and 2B10 (F) $(n=51)$. 